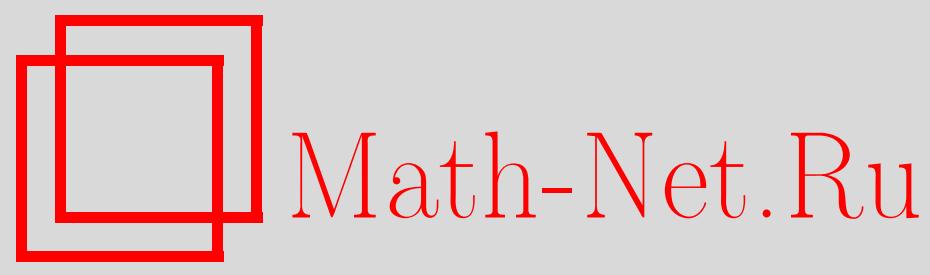

Б. С. Кашин, А. А. Разборов, Новые нижние оценки устойчивости матриц Адамара, Матем. заметки, 1998, том 63, выпуск 4, 535-540

DOI: https://doi.org/10.4213/mzm1314

Использование Общероссийского математического портала Math-Net.Ru подразумевает, что вы прочитали и согласны с пользовательским соглашением http://www . mathnet.ru/rus/agreement

Параметры загрузки:

IP : 54.209 .52 .79

26 апреля 2023 г., 13:02:55

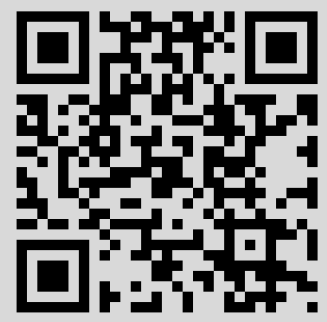




\title{
НОВЫЕ НИЖНИЕ ОЦЕНКИ УСТОЙЧИВОСТИ МАТРИЦ АДАМАРА
}

\author{
Б. С. Кашин, А. А. Разборов
}

Пусть соотношение $f=\Omega(g)$ означает, что $f(x) \geqslant c g(x)$ для некоторой положительной постоянной $c$ и для всех $x$ из области определения функций $f$ и $g$. Показано, что в произвольной (обобщенной) матрице Адамара необходимо изменить по крайней мере $\Omega\left(n^{2} / r\right)$ элементов, для того чтобы ранг полученной матрицы не превосходил $r$. Это улучшает ранее известную оценку $\Omega\left(n^{2} / r^{2}\right)$. Если дополнительно потребовать, чтобы измененные элементы были ограничены сверху по модулю некоторой величиной $\theta \geqslant n / r$, то справедлива другая оценка $\Omega\left(n^{3} /\left(r \theta^{2}\right)\right)$, усиливающая предыдущую $\Omega\left(n^{2} / \theta^{2}\right)$.

Библиография: 20 названий.

1. Введение. Для поля $k$ и матрицы $A$ над этим полем обозначим через $R_{A}^{k}(r) \phi y н к-$ иию устойчивости $A$, определяемую как минимальное количество изменений элементов матрицы $A$, необходимое для получения матрицы ранга не вьше $r$. Это обозначение ввел Валиант [1], [2]; независимо сходное понятие предложил Григорьев [3].

Основной причиной введения и изучения этого понятия является то обстоятельство, что устойчивые матрицы являются сложньпи по отношению ко многим важным вычислительньм моделям, получение нижних оценок для которых - трудная задача, не решенная к настоящему времени. Такими моделями являются, например, линейные алгебраические схемы [2], [4] и вычисления с ограниченньп числом альтераций в теории коммуникационной сложности [5], [6]. В частности, для любого конкретного примера достаточно устойчивой матрищы можно было бы построить соответствующую вычислительную задачу, являющуюся сложной с точки зрения указанных моделей. Это привело бы к существенным продвижениям в этой области.

Несмотря на многочисленные усилия, наилучшие известные нижние оценки величины $R_{A}^{k}(r)$, полученные для конкретных $(n \times n)$-матриц $A$, имеют вид $\Omega\left(n^{2} /(r \log (n / r))\right)$ для конечного поля $k$ (Фридман [7]) и $\Omega\left(n^{2} / r\right)$ в случае бесконечного поля $k(r \leqslant n / 2)$. Простейший способ получения последней оценки был независимо отмечен Григорьевьм и Нисаном: для любой вполне невырожденной матрицы $A$ (т.е. матрицы, все миноры которой невырожденны) вьполнено $R_{A}^{k}(r) \geqslant \Omega\left(n^{2} / r\right)(k$ произвольно, $r \leqslant n / 2)$. Другие методы получения подобной оценки $\Omega\left(n^{2} / r\right)$, реализованные для различных классов

Работа первого автора выполнена при поддержке Российского фонда фундаментальных исследований, гранты № 96-01-00094 и № 96-15-96102, и фонда INTAS, грант № 93-1376. Работа второго автора выполнена при поддержке Российского фонда фундаментальных исследований, гранты № 96-01-01222 и № 96-15-96090. 
матриц, были предложены в [5], [8]-[10]; некоторые из них применимы и в случае конечных полей.

Одним из классов матриц, для которого естественно предполагать наличие свойства сильной устойчивости над $\mathbb{R}$, является класс матрищ Адамара. Эти матрищы хорошо приспособлены к изучению спектральными методами, которые зарекомендовали себя как чрезвычайно удобньй инструмент для решения подобных задач в теории сложности (см. типичные примеры в [11], [12]). Пудлак, Разборов и Савицкий [13] доказали оценку $R_{H}^{\mathbb{R}}(r) \geqslant \Omega\left(n^{2} /\left(r^{3} \log r\right)\right)$, где $H$-произвольная матрища Адамара, а Алон [14] усилил ее: $R_{H}^{\mathbb{R}}(r) \geqslant \Omega\left(n^{2} / r^{2}\right)$. Исследуя упомянутые выше приложения в теории коммуникационной сложности, Локам [6] рассмотрел модифицированную функцию устойчивости $R_{A}^{\mathbb{C}}(r, \theta)$, соответствующую случаю, когда дополнительно требуется, чтобы абсолютные величины измененных элементов не превосходили $\theta$, и показал, в частности, что $R_{H}^{\mathbb{C}}(n / 2, \theta) \geqslant \Omega\left(n^{2} / \theta^{2}\right)$ для любой (обобщенной) матрицы Адамара $H$.

В настоящей заметке мы устанавливаем усиления некоторых из упомянутых выше результатов. Именно, для произвольной (обобщенной) матрицы Адамара $H$ и любого $r \leqslant n / 2$ мы доказьваем, что $R_{H}^{\mathbb{C}}(r) \geqslant \Omega\left(n^{2} / r\right)$, и если $\theta$ является параметром, удовлетворяющим неравенству $\theta \geqslant n / r$, то $R_{H}^{\mathbb{C}}(r, \theta) \geqslant \Omega\left(n^{3} /\left(r \theta^{2}\right)\right)$. Наши оценки слишком слабые, чтобы получить какие-либо содержательные следствия в теории сложности, и они не переходят гранищу $n^{2} / r$. Основная цель настоящей заметки - привлечь внимание специалистов к использованию в этой области спектральньх методов, так как мы уверены, что их потенциал далеко не исчерпан.

\section{2. Устойчивость матриц.}

ОПРеДЕЛЕниЕ 1 [1], [2], [6]. Для матрицы $A$ над некоторьм полем $k$ обозначим через wt $(A)$ количество ее ненулевых элементов. Функиия устойчивости $R_{A}^{k}(r)$ матрицы $A$ определяется по формуле

$$
R_{A}^{k}(r) \rightleftharpoons \min _{B}\{\mathrm{wt}(A-B) \mid \operatorname{rk}(B) \leqslant r\}
$$

где $B$ пробегает все матрицы того же размера, что и $A$. Если $k=\mathbb{C}$ и $\theta-$ положительное вещественное число, то положим

$$
R_{A}^{\mathbb{C}}(r, \theta) \rightleftharpoons \min _{B}\left\{\operatorname{wt}(A-B)|\operatorname{rk}(B) \leqslant r,| a_{i j}-b_{i j} \mid \leqslant \theta \forall i, j\right\} .
$$

ЗАмЕЧАНИЕ 1. Локам [6] использовал в своем определении функции $R_{A}^{\mathbb{C}}(r, \theta)$ ограничение $\left|b_{i j}\right| \leqslant \theta$ вместо нашего $\left|a_{i j}-b_{i j}\right| \leqslant \theta$. Однако, более естественньп представляется оценивать $\ell_{\infty}$-норму изменений, а не конечного результата. В частности, наше определение позволяет также устанавливать нетривиальные оценки в случае $\theta \ll 1$ (см. ниже предложение 1).

ОПРЕДЕЛЕНИЕ 2. Комплексная $(n \times n)$-матрица $H$ называется обобщенной матричей Адамара, если $\left|h_{i j}\right|=1$ для всех $i, j$ и $H H^{*}=n I_{n}$, где $H^{*}-$ сопряженная к $H$ матрица и $I_{n}$ - единичная $(n \times n)$-матрища.

ПРЕДЛОЖЕНИЕ 1. ДЛя любой обобщенной $(n \times n)$-матрицы Адамара $H$ и любого $r \leqslant n / 2$
a) $R_{H}^{\mathbb{C}}(r) \geqslant \Omega\left(n^{2} / r^{2}\right)($ c.M. [14], [6]);
б) $R_{H}^{\mathbb{C}}(n / 2, \theta) \geqslant \Omega\left(n^{2} / \theta^{2}\right)$, әде $\theta>0-$ произвольныи параметр (см. [6]);
в) $R_{H}^{\mathbb{C}}(n / 2, \theta) \geqslant \Omega\left(n^{2} / \theta\right)$ в случае $\theta \leqslant n / r($ с.м. $[6])$. 
ЗАМЕчАнИЕ 2. Строго говоря, предложение 1в) сформулировано в [6] для более ограничительного варианта устойчивости $R_{A}^{+}(r, \theta)$, получающегося добавлением в (1) дополнительного условия $\left|b_{i j}\right| \geqslant 1$. Однако, несложный анализ доказательства Локама показывает, что это условие может быть опущено.

В настоящей заметке мы докажем, что справедлива

ТЕОремА. Для любой обобщенной $(n \times n)$-матрицы Адамара $H$ и любого $r \leqslant n / 2$

a) $R_{H}^{\mathbb{C}}(r) \geqslant \Omega\left(n^{2} / r\right)$;

б) $R_{H}^{\mathbb{C}}(r, \theta) \geqslant \Omega\left(n^{3} /\left(r \theta^{2}\right)\right)$ в случае $\theta \geqslant n / r$.

Утверждение б) теоремы усиливает предложение 1в) на множитель $n / r$ при $\theta \geqslant n / r$ (его формулировка и доказательство могут быть рассмотрены как "непрерьвное продолжение" предложения 1в) при $\theta \geqslant n / r)$.

3. Спектры матриц. Норма Фробениуса $\|A\|_{F}$ комплексной матрицы $A$ определяется, как

$$
\|A\|_{F} \rightleftharpoons\left(\sum_{i, j}\left|a_{i j}\right|^{2}\right)^{1 / 2} .
$$

Пусть $(n \times n)$-матрица $A$ является вещественной симметричной и $\lambda_{1}(A) \geqslant \cdots \geqslant \lambda_{n}(A)$ - ее (вешественные) собственные значения. Тогда

$$
\begin{aligned}
& \operatorname{Tr}(A)=\lambda_{1}(A)+\cdots+\lambda_{n}(A), \\
& \|A\|_{F}^{2}=\lambda_{1}^{2}(A)+\cdots+\lambda_{n}^{2}(A) .
\end{aligned}
$$

Пусть $r=\operatorname{rk}(A)$, т.е. в точности $r$ собственньх значений среди $\lambda_{1}(A), \ldots, \lambda_{n}(A)$ ненулевые. Из неравенства Коши согласно (2) и (3) следует, что $\operatorname{Tr}(A)^{2} \leqslant\|A\|_{F}^{2} \cdot r$. Таким образом, справедлива

ЛЕмма 1. Для любой вещественной симметричной матрицы $A$

$$
\operatorname{rk}(A) \geqslant \frac{\operatorname{Tr}(A)^{2}}{\|A\|_{F}^{2}} .
$$

Напомним также неравенство Хоффмана-Виландта. Через $\sigma_{i}(A) \rightleftharpoons \sqrt{\lambda_{i}\left(A A^{*}\right)}$ обозначим $i$-е сингулярное число комплексной матрицы $A$.

ПРЕДЛОЖЕНИЕ 2 [15], [16, разд. 8.3]. Для любой пары комплексных $(n \times n)$-матpuи, $A, B$

$$
\sum_{i=1}^{n}\left(\sigma_{i}(A)-\sigma_{i}(B)\right)^{2} \leqslant\|A-B\|_{F}^{2} .
$$

4. Доказательство теоремы. а) Как и в предшествующих доказательствах (см. [13], [14], [6]), мы будем стремиться показать, что подматрицы произвольной обобщенной матрищы Адамара имеют достаточно высокий ранг (по крайней мере в среднем), а это позволит перенести на наш случай наблюдение Григорьева и Нисана для вполне невырожденных матриц.

В действительности, в функциональном анализе имеется ряд результатов (см., в частности, [17]-[20]), посвященных общему вопросу: “насколько ортогональны выбранные 
случайным образом подматрицы ортогональной матрищы”? Поскольку “достаточно ортогональные" матрицы являются невырожденньми, мы можем непосредственно применить результаты из [19], [20] для получения оценки $R_{H}^{\mathbb{C}}(r) \geqslant \Omega\left(n^{2} /(r \log n)\right)$. С другой стороны, для наших целей необходимо нечто более слабое, чем то, что дает достаточно тонкая техника из [19], [20]. Поэтому мы заменим ее следуюшей простой леммой, позволяющей избавиться от логарифмического множителя в знаменателе.

Всюду далее мы используем жирньй шрифт для обозначения случайных объектов.

Лемма 2. Пусть $q \leqslant n u A-$ комплексная $(q \times n)$-матрииа такая, что $\left|a_{i j}\right|=1$ для всех $i, j u A A^{*}=n I_{q}$. Пусть $\boldsymbol{B}$ - случайно выбранная $(q \times q)$-подматрица матрицы A. Тогда для любого $r$

$$
\mathrm{P}[\mathrm{rk}(\boldsymbol{B}) \leqslant r] \leqslant \frac{2 r}{q} .
$$

ДокАЗАТЕЛЬСтво. Обозначим $\boldsymbol{B} \boldsymbol{B}^{*}$ через $\boldsymbol{C}$. Тогда $\boldsymbol{C}$ является (положительно определенной $)$ вещественной симметричной $(q \times q)$-матрицей, все элементы которой на главной диагонали равны $q$ (поэтому $\operatorname{Tr}(\boldsymbol{C})=q^{2}$ ) и которая удовлетворяет неравенству $\operatorname{rk}(\boldsymbol{C}) \leqslant \operatorname{rk}(\boldsymbol{B})$. Таким образом, в силу леммы 1 (примененной к $A:=\boldsymbol{C}$ )

$$
\operatorname{rk}(\boldsymbol{B}) \leqslant r \Longrightarrow\|\boldsymbol{C}\|_{F}^{2} \geqslant \frac{q^{4}}{r} .
$$

С другой стороны, согласно неравенству Чебьшева

Положим

$$
\mathrm{P}\left[\|\boldsymbol{C}\|_{F}^{2} \geqslant \frac{q^{4}}{r}\right] \leqslant \frac{r}{q^{4}} \cdot \mathrm{E}\left[\|C\|_{F}^{2}\right] .
$$

$$
\boldsymbol{\xi}_{j} \rightleftharpoons \begin{cases}1, & \text { если } j \text {-й столбец представлен в матрище } \boldsymbol{B}, \\ 0 & \text { в противном случае; }\end{cases}
$$

случайный вектор $\left(\boldsymbol{\xi}_{1}, \ldots, \boldsymbol{\xi}_{n}\right)$ равномерно распределен на множестве всех векторов в $\{0,1\}^{n}$, имеющих ровно $q$ единиц. Пусть $\boldsymbol{c}_{i_{1} i_{2}}=\sum_{j=1}^{n} a_{i_{1} j} a_{i_{2} j} \boldsymbol{\xi}_{j}$. Тогда

$$
\begin{aligned}
\|\boldsymbol{C}\|_{F}^{2} & =\sum_{i_{1}, i_{2}}\left(\boldsymbol{c}_{i_{1} i_{2}} \boldsymbol{c}_{i_{1} i_{2}}^{*}\right)=\sum_{i_{1}, i_{2}} \sum_{j_{1}, j_{2}}\left(a_{i_{1} j_{1}} a_{i_{2} j_{1}} a_{i_{1} j_{2}}^{*} a_{i_{2} j_{2}}^{*} \boldsymbol{\xi}_{j_{1}} \boldsymbol{\xi}_{j_{2}}\right) \\
& =\sum_{j_{1}, j_{2}}\left(\sum_{i_{1}, i_{2}} a_{i_{1} j_{1}} a_{i_{2} j_{1}} a_{i_{1} j_{2}}^{*} a_{i_{2} j_{2}}^{*}\right) \boldsymbol{\xi}_{j_{1}} \boldsymbol{\xi}_{j_{2}} .
\end{aligned}
$$

Далее

$$
\mathrm{E}\left[\boldsymbol{\xi}_{j_{1}} \boldsymbol{\xi}_{j_{2}}\right]= \begin{cases}\frac{q}{n}, & \text { если } j_{1}=j_{2}, \\ \frac{q(q-1)}{n(n-1)}, & \text { если } j_{1} \neq j_{2} .\end{cases}
$$

Следовательно,

$$
\begin{aligned}
\mathrm{E}\left[\|\boldsymbol{C}\|_{F}^{2}\right]= & \frac{q(q-1)}{n(n-1)} \sum_{j_{1}, j_{2}} \sum_{i_{1}, i_{2}}\left(a_{i_{1} j_{1}} a_{i_{2} j_{1}} a_{i_{1} j_{2}}^{*} a_{i_{2} j_{2}}^{*}\right) \\
& +\left(\frac{q}{n}-\frac{q(q-1)}{n(n-1)}\right) \sum_{j} \sum_{i_{1}, i_{2}}\left(a_{i_{1} j} a_{i_{2} j} a_{i_{1} j}^{*} a_{i_{2} j}^{*}\right) \\
= & \frac{q(q-1)}{n(n-1)}\left\|A A^{*}\right\|_{F}^{2}+\left(\frac{q}{n}-\frac{q(q-1)}{n(n-1)}\right) n q^{2}=q^{2}\left(q+\frac{q-1}{n-1}(n-q)\right) \leqslant 2 q^{3} .
\end{aligned}
$$

Теперь лемма 2 непосредственно вытекает из (4)-(6). 
СлЕДСТВИЕ. Пусть $H$ - обобщенная $(n \times n)$-матрица Адамара и $\boldsymbol{H}_{0}-$ ее случайная $(q \times q)$-подматрица. Тогда $\mathrm{E}\left[\operatorname{rk}\left(\boldsymbol{H}_{0}\right)\right] \geqslant q / 8$.

ДокАЗАТЕЛЬСТво. Подматрица $\boldsymbol{H}_{0}$ строится в два этапа: сначала мы выбираем случайным образом $q$ строк, затем $q$ столбцов. Поскольку $(q \times n)$-матрица $\boldsymbol{A}$, получающаяся на первом этапе, удовлетворяет требованиям леммы 2 , для любого фиксированного выбора строк $1 \leqslant i_{1}<\cdots<i_{q} \leqslant n$ имеет место оценка условной вероятности $\mathrm{P}\left[\operatorname{rk}\left(\boldsymbol{H}_{0}\right) \leqslant q / 4 \mid \boldsymbol{H}_{0}\right.$ содержит строки $\left.i_{1}, \ldots, i_{q}\right] \leqslant 1 / 2$. Следовательно, $\mathrm{P}\left[\operatorname{rk}\left(\boldsymbol{H}_{0}\right) \leqslant\right.$ $q / 4] \leqslant 1 / 2$ и $\mathrm{E}\left[\operatorname{rk}\left(\boldsymbol{H}_{0}\right)\right] \geqslant q / 4 \cdot \mathrm{P}\left[\operatorname{rk}\left(\boldsymbol{H}_{0}\right) \geqslant q / 4\right] \geqslant q / 8$.

Теперь мы готовы завершить доказательство теоремы. Пусть $H$ - обобщенная $(n \times n)$-матрица Адамара и $r \leqslant n / 2$. Поскольку оценка $R_{H}^{\mathbb{C}}(n / 2) \geqslant n / 2$ очевидна, не ограничивая общности можем считать, что $r \leqslant n / 16$. Пусть матрища $A$ такова, что $\operatorname{rk}(H-A) \leqslant r$. Положим $q=16 r$ и в матрице $H$ случайным образом выберем $(q \times q)$-подматрицу $\boldsymbol{H}_{0}$. Если $\boldsymbol{A}_{0}-$ соответствующая подматрица матрищы $A$, то

$$
\mathrm{E}\left[\operatorname{rk}\left(\boldsymbol{H}_{0}\right)\right] \leqslant \mathrm{E}\left[\operatorname{rk}\left(\boldsymbol{H}_{0}-\boldsymbol{A}_{0}\right)\right]+\mathrm{E}\left[\operatorname{rk}\left(\boldsymbol{A}_{0}\right)\right] \leqslant r+\mathrm{E}\left[\operatorname{wt}\left(\boldsymbol{A}_{0}\right)\right]=r+\frac{q^{2}}{n^{2}} \operatorname{wt}(A) .
$$

$\mathrm{C}$ другой стороны, $\mathrm{E}\left[\operatorname{rk}\left(\boldsymbol{H}_{0}\right)\right] \geqslant 2 r$ согласно следствию из леммы 2 . Объединяя два последних неравенства, заключаем, что $\operatorname{wt}(A) \geqslant r n^{2} / q^{2}=\Omega\left(n^{2} / r\right)$. Это завершает доказательство утверждения а) теоремы.

б) Предложение 1в) было доказано в [6] путем применения неравенства ХоффманаВиландта к паре матриц $H, \frac{1}{\theta} B$. Мы обобщим этот подход на случай $\theta \geqslant n / r$ варьированием коэффициента при матрище $B$.

Пусть, как и ранее, $H$ - обобщенная $(n \times n)$-матрица Адамара и $r \leqslant n / 2$. Пусть матрица $A$ такова, что $\operatorname{rk}(H-A) \leqslant r$ и $\left|a_{i j}\right| \leqslant \theta$ для всех $i, j$, где $\theta \geqslant n / r-$ еще один параметр. Применяя предложение 2 к паре матриц $H, \frac{r}{n}(H-A)$, мы, как и в [6], имеем

$$
\left\|H-\frac{r}{n}(H-A)\right\|_{F}^{2} \geqslant n(n-r) .
$$

С другой стороны,

$$
H-\frac{r}{n}(H-A)=\left(1-\frac{r}{n}\right) H+\frac{r}{n} A .
$$

Следовательно, эта матрица имеет не более $\operatorname{wt}(A)$ элементов, ограниченных по модулю величиной $1+r \theta / n$, в то время как остальные элементы по модулю не превосходят $1-r / n$. Отсюда следует оценка

$$
\left\|H-\frac{r}{n}(H-A)\right\|_{F}^{2} \leqslant \operatorname{wt}(A)\left(1+\frac{r \theta}{n}\right)^{2}+\left(1-\frac{r}{n}\right)^{2} n^{2} .
$$

Из неравенств (7) и (8) получаем

$$
\mathrm{wt}(A)\left(1+\frac{r \theta}{n}\right)^{2} \geqslant r(n-r) .
$$

Поскольку $r \leqslant n / 2$ и $\theta \geqslant n / r$, отсюда следует требуемая оценка $\operatorname{wt}(A) \geqslant \Omega\left(n^{3} /\left(r \theta^{2}\right)\right)$. Это завершает доказательство утверждения б) теоремы. 


\section{СПИСОК ЦИТИРОВАННОЙ ЛИТЕРАТУРЫ}

[1] Valiant L. G. Some Conjectures Relating to Superlinear Complexity Bounds. Tech. Report no. 85: Univ. of Leeds, 1976.

[2] Valiant L. G. Graph-Theoretic Arguments in Low-Level Complexity. Tech. Report no. 13-77: Univ. of Edinburgh, Dept. of Comp. Sci., 1977.

[3] Григорьев Д. Ю. Использование понятий отделенности и независимости для получения нижних оценок сложности схем // Записки научн. семин. ЛОМИ. 1976. Т. 60. С. 38-48.

[4] Григорьев Д. Ю. Нижние оценки в алгебраической сложности вычислений // Записки научн. семин. ЛОМИ. 1982. Т. 118. С. 25-82.

[5] Разборов А. А. Об устойчивых матрицах. Препринт. М.: МИАН, 1989.

[6] Lokam S. V. Spectral methods for matrix rigidity with applications to size-depth tradeoffs and communication complexity // Proc. of the 36th IEEE Symposium on Foundations of Computer Science. Los Alamitos (C.A.): IEEE, 1995. P. 6-15.

[7] Friedman J. A note on matrix rigidity // Combinatorica. 1993. V. 13. № 2. P. 235-239.

[8] Pudlák P., Vavřín Z. Computation of rigidity of order $n^{2} / r$ for one simple matrix // Comment. Math. Univ. Carolin. 1991. V. 32. № 2. P. 213-218.

[9] Kimmel P., Settle A. Reducing the Rank of Lower Triangular All-Ones Matrix. Tech. Report CS 92-21: Univ. of Chicago, 1992.

[10] Pudlák P. Large communication in constant depth circuits // Combinatorica. 1994. V. 14. № 2. P. 203-216.

[11] Krause M., Waack S. Variation ranks of communication matrices and lower bounds for depth two circuits having symmetric gates with unbounded fan-in // Proc. of the 32nd IEEE Symposium on Foundations of Computer Science. Los Alamitos (C.A.): IEEE, 1991. P. 777-782.

[12] Nisan N., Wigderson A. On the complexity of bilinear forms // Proceedings of the 27th ACM Symposium on the Theory of Computing. New York: ACM, 1995. P. 723-732.

[13] Pudlák P., Razborov A., Savický P. Observations on Rigidity of Hadamard Matrices. Manuscript, 1988.

[14] Alon N. On the Rigidity of Hadamard Matrices. Manuscript: Tel Aviv Univ., 1990.

[15] Hoffman A. J., Wielandt H.W. The variation of the spectrum of a normal matrix // Duke Math. J. 1953. V. 20. P. 37-39.

[16] Golub G. H., van Loan C. F. Matrix Computations. Baltimore, Maryland: John Hopkins Univ. Press, 1983.

[17] Кашин Б. С. О некоторых свойствах матриц ограниченных операторов из пространства $\ell_{2}^{n}$ в $\ell_{2}^{m}$ // Изв. АН АрмССР. Матем. 1980. Т. 15. № 5. С. 379-394.

[18] Лунин А.А. Об операторных нормах подматриц // Матем. заметки. 1989. Т. 45. № 3. C. $94-100$.

[19] Kashin B., Tzaffiri L. Some Remarks on the Restriction of Operators to Coordinate Subspaces. Tech. Report no. 12. Jerusalem: The Edmund Landau Center for Research in Math. Anal., Hebrew Univ., 1993/94.

[20] Rudelson M. Almost orthogonal submatrices of an orthogonal matrix // Israel J. Math. (to appear).

Математический институт им. В. А. Стеклова РАН

Поступило

E-mail: kashin@mi.ras.ru, razborov@mi.ras.ru 